\title{
The genome sequence of Brucella pinnipedialis B2/94 sheds light on the evolutionary history of the genus Brucella
}

\author{
Stéphane Audic ${ }^{1,2,3^{*}}$, Magali Lescot ${ }^{1}$, Jean-Michel Claverie ${ }^{1}$, Axel Cloeckaert ${ }^{4}$ and Michel S Zygmunt ${ }^{4 *}$
}

\begin{abstract}
Background: Since the discovery of the Malta fever agent, Brucella melitensis, in the 19th century, six terrestrial mammal-associated Brucella species were recognized over the next century. More recently the number of novel Brucella species has increased and among them, isolation of species B. pinnipedialis and B. ceti from marine mammals raised many questions about their origin as well as on the evolutionary history of the whole genus.

Results: We report here on the first complete genome sequence of a Brucella strain isolated from marine mammals, Brucella pinnipedialis strain B2/94. A whole gene-based phylogenetic analysis shows that five main groups of host-associated Brucella species rapidly diverged from a likely free-living ancestor close to the recently isolated $B$. microti. However, this tree lacks the resolution required to resolve the order of divergence of those groups. Comparative analyses focusing on a) genome segments unshared between B. microti and B. pinnipedialis, b) gene deletion/fusion events and c) positions and numbers of Brucella specific IS711 elements in the available Brucella genomes provided enough information to propose a branching order for those five groups.

Conclusions: In this study, it appears that the closest relatives of marine mammal Brucella sp. are B. ovis and Brucella sp. NVSL 07-0026 isolated from a baboon, followed by B. melitensis and B. abortus strains, and finally the group consisting of $B$. suis strains, including B. canis and the group consisting of the single B. neotomae species. We were not able, however, to resolve the order of divergence of the two latter groups.
\end{abstract}

Keywords: Brucella, Bacterial Genome Evolution, Comparative Genomics

\section{Background}

Brucellae are Gram-negative, facultative, intracellular bacteria that can infect many species of animals and man. Six species were initially recognized within the genus Brucella: B. abortus, B. melitensis, B. suis, B. ovis, B. canis, and B. neotomae [1-3]. This classification is mainly based on differences in pathogenicity, host preference, and phenotypic characteristics. Four additional species have been included in the genus Brucella since 2007. These comprise the species B. ceti and B. pinnipedialis

\footnotetext{
* Correspondence: stephane.audic@sb-roscoff.fr; Michel.Zygmunt@tours.inra.fr 'Laboratoire Information Génomique et Structurale, CNRS-UPR2589, AixMarseille University, Institut de Microbiologie de la Méditerranée (IM2, IFR88), Parc Scientifique de Luminy-163 Avenue de Luminy-Case 934-FR-13288, Marseille cedex 09, France

${ }^{4}$ INRA, UR1282, Infectiologie Animale et Santé Publique, IASP, Nouzilly, F37380, France

Full list of author information is available at the end of the article
}

isolated from marine mammals, with cetaceans (dolphin, porpoise, and whale species) and pinnipeds (various seal species) as preferred host respectively $[4,5]$. B. microti described in 2008 was first isolated from the common vole and then from the red fox, and from soil [6-8]. The latest described species is $B$. inopinata, isolated from an infected human breast implant, and currently the most divergent Brucella species at the phenotypic and molecular level $[9,10]$. The animal or environmental reservoir of B. inopinata is not known. New Brucella species will likely be described in the future such as for isolates from baboons [11], from wild rodents in Australia [12] and for strain $\mathrm{BO} 2$ isolated from a patient with chronic destructive pneumonia [13]. Strain $\mathrm{BO} 2$ and strains from wild Australian rodents have been proposed as a novel lineage of the B. inopinata species $[12,13]$.
C Biomed Central

C 2011 Audic et al; licensee BioMed Central Ltd. This is an Open Access article distributed under the terms of the Creative Commons Attribution License (http://creativecommons.org/licenses/by/2.0), which permits unrestricted use, distribution, and reproduction in any medium, provided the original work is properly cited. 
Molecular and phenotypic typing of marine mammal Brucella strains led to their classification into two species, $B$. ceti and B. pinnipedialis, according to their preferred host, cetaceans and pinnipeds respectively [5]. However, several subgroups were identified within each species by molecular typing methods such as multilocus sequence analysis (MLSA), multilocus VNTR (Variable Number of Tandem Repeats) analysis (MLVA), or omp2a and omp $2 b$ porin genes [14-19]. Among them one subgroup within $B$. ceti, exclusively composed of strains isolated from various dolphin species, was proposed to constitute a separate species with the name $B$. delphini $[3,14,18]$. The isolates from cetaceans from the Pacific may also constitute a separate species [19]. Three human cases with naturally acquired infection by Brucella strains presumably from marine origin were reported, one case of spinal osteomyelitis from a patient in New Zealand [20] and two neurobrucellosis cases from Peruvian patients [21]. Interestingly, these human isolates exhibited the same genotype as strains from cetaceans from the Pacific [22].

Among their distinctive characteristics at the molecular and genomic level, marine mammal Brucella strains were shown to carry in their genomes a higher number of the insertion sequence element IS711 (or IS6501) [23,24] than terrestrial mammal Brucella species and biovars with the exception of B. ovis $[15,17,25]$. Consequently, infrequent restriction site-PCR (IRS-PCR) methods and more recently ligation-mediated PCR (LM-PCR) were applied, taking into account this higher number of IS711 elements, to study the genomic diversity of marine mammal strains [26-28]. These studies confirmed the classification into two marine mammal Brucella species, each divided in subgroups. In addition, six specific IS711-containing DNA fragments were detected allowing the molecular identification of $B$. ceti and its subgroup composed exclusively of dolphin isolates [17,26-28]. Besides these specific IS711containing fragments another DNA fragment was detected that was exclusively found in B. pinnipedialis strains, with the exception of hooded seal isolates, consisting of a putative genomic island [26,27]. The size of this island was estimated at $62 \mathrm{kbp}$ according to the physical maps made from the genomes of marine mammal strains by macrorestriction analyses [14].

The taxonomy of Brucella is still controversial, with an ongoing debate on whether they should be considered as distinct species or distinct strains of B. melitensis, considering the close proximity of their genomes [29]. We determined and analyzed the complete genome of $B$. pinnipedialis $\mathrm{B} 2 / 94$ to bring new insights into the origin of Brucella isolated from marine mammals as well as their time of divergence from Brucella isolated from terrestrial animals.

\section{Results and Discussion}

The genome sequence of $B$. pinnipedialis B2/94 was determined $(30 \times$ coverage) by shotgun sequencing with the GS-FLX technology and the remaining gaps filled using the standard Sanger technology. Like that of other Brucella strains, the genome is composed of two circular chromosomes, of 2,138,342 bp (base-pairs) and $1,260,926 \mathrm{bp}$ in lengths, respectively. Bioinformatic annotation predicted the presence of 3,342 protein coding genes, 55 tRNAs and 9 ribosomal RNAs. The comparison with the known genomes of other Brucella species revealed the presence of 90 pseudogenes. The $23 \mathrm{~S}$ rDNA sequence of $B$. pinnipedialis B2/94 was found similar to that of other Brucella species, in contrast with the anomalous and unexpected 23S ribosomal RNA sequence previously described for B. microti [30].

\section{Genome structure and whole gene set phylogeny}

The phylogenetic placement of B. pinnipedialis B2/94 was assessed with the help of a whole gene tree including all orthologous genes from Brucella strains available in the complete genome division of GenBank, completed by sequence data from selected Brucella strains of particular phylogenetic relevance, either deposited in the whole genome shotgun (WGS) division of GenBank, or available from other sources (see Methods). This includes sequences from $B$. neotomae, 10 sequences from marine mammal Brucella strains, Brucella sp. B02, B. inopinata BO1, Brucella sp. 83/13 or Brucella sp. NF2653. The resulting tree (Figure 1 ) readily shows that the Brucella species with the highest sequence similarity with $B$. pinnipedialis $\mathrm{B} 2 / 94$ and whose complete genome is available is $B$. microti CCM 4915. This is a consequence of the relatively slow evolution rate of those two species rather than reflecting a particular phylogenetic affinity The central position occupied by B. microti, just at the root of host-associated Brucella species, is also confirmed. This tree distinguishes five major groups, the $B$. suis strains (shaded rectangle A, Figure 1 ), the different $B$. melitensis and $B$. abortus strains, also well separated from each other (rectangle B), another group with B. ovis and Brucella sp. NVSL 07-0026 (rectangle C), and marine mammal Brucella strains (rectangle D). B. neotomae remains isolated in this tree and constitutes the fifth group. However, the resolution of this tree is insufficient to infer the relative order of divergence of those different groups. Moreover, while bootstrap values in this tree are in general very strong (99 or 100\%), the branching $(D,(C$, B)) proposed in the maximum likelihood tree is generally not supported, with a bootstrap value of $12 \%$, prompting for a finer analysis of the events shaping the Brucella evolutionary tree. 


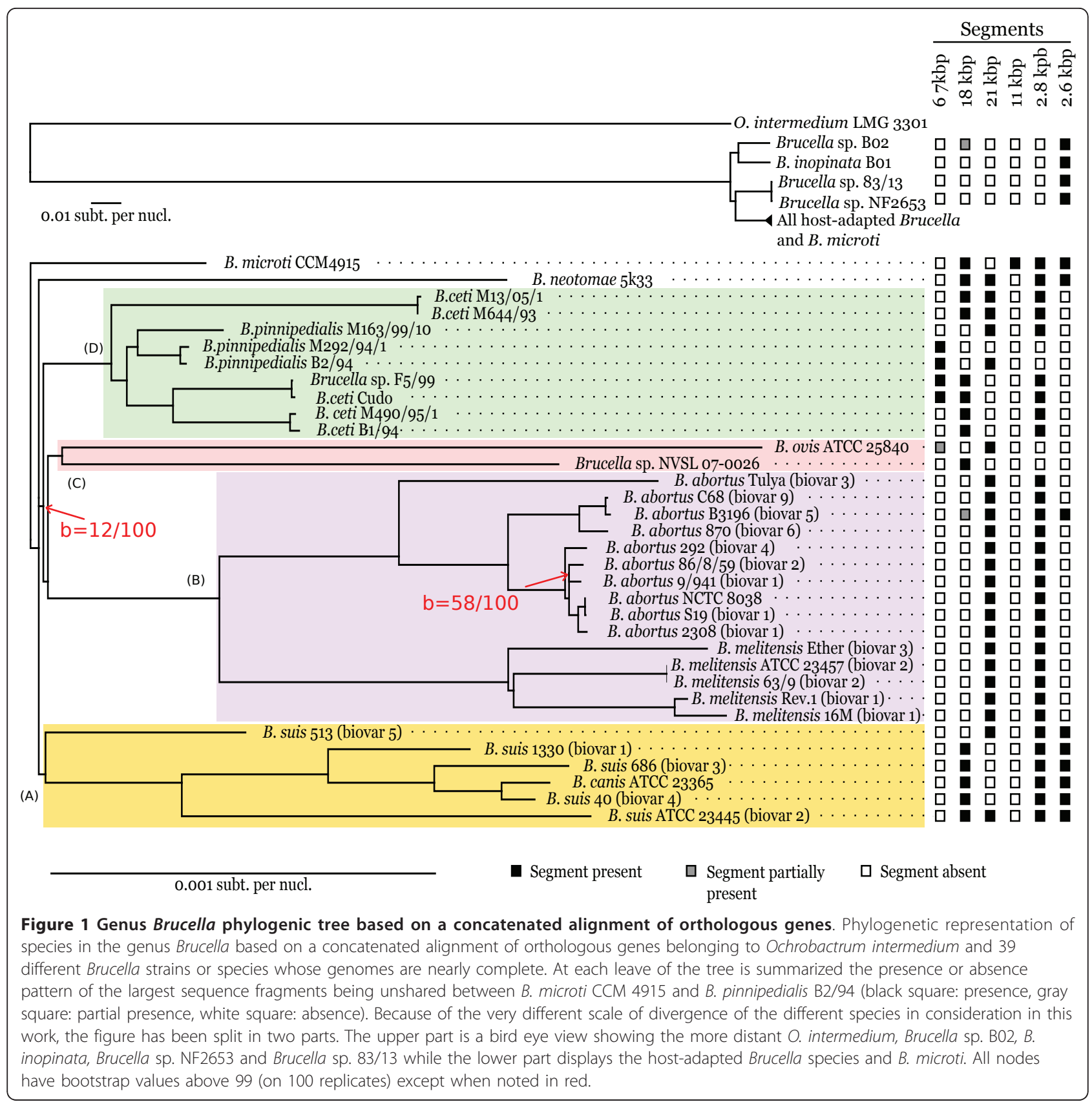

The genome sequences of B. microti CCM 4915 and B. pinnipedialis $\mathrm{B} 2 / 94$ were found to be remarkably conserved even at the nucleotide level, allowing to generate a complete alignment of the chromosomes of the two species from which a list of all indels (insertions and deletions) (available as Additional file 1, Table S1 and Additional file 2, Table S2) was easily obtained. In spite of the overall similarity of these two genomes, this alignment revealed major changes in genome structure, as large segments unshared between the two species. The alignment from the largest chromosome exhibited 238 gapped positions with the largest insert in $B$. microti being 2,653 bp long, and the largest in $B$. pinnipedialis being 21,713 bp long. The alignment from the small chromosome exhibits 151 gapped positions, the largest insert being 18,341 bp long in B. microti and 67,389 bp long in B. pinnipedialis. The total number of indels (389) found in comparing these two species is thus smaller than the 405 indels found in comparing B. microti CCM 4915 and $B$. suis 1330 [30]. The complete alignment contains a total of 3,290,621 aligned positions (2,104,923 and $1,185,698$ per chromosome), with $0.10 \%$ of nucleotide 
changes in aligned regions (2,195 and 1,200 nucleotide changes, respectively). This fraction of nucleotide substitution is also smaller than what was observed between B. microti CCM 4915 and B. suis 1330 , where a $0.16 \%$ divergence was reported [30].

\section{Detailed analysis of the largest unshared sequence regions and the branching order of host associated Brucella species}

The evolutionary history of the largest segments unshared between B. microti CCM 4915 and B. pinnipedialis B2/94 was analyzed by examining the structure of the orthologous loci in the other Brucella species for which sequence data was available (see Methods). On the large chromosome, the 2 largest indels are $21 \mathrm{kbp}$, and $2.6 \mathrm{kbp}$ in length. On the small chromosome, the largest indels are $67 \mathrm{kbp}, 18 \mathrm{kpb}, 11 \mathrm{kbp}$ and $2.8 \mathrm{kbp}$ in length. All other indels are at most the size of an IS711 insertion sequence (843 bp). The presence/absence of the above genomic inserts was assessed in other Brucella strains (see Methods). For each segment, we recorded the number of nucleotides with homologues in the other Brucella genomes (Additional file 3, Table S3). The presence/absence of those segments is reported at the leaves of the tree (Figure 1) as filled squares. The evolutionary history of those unshared genome segments, treated as discrete characters, was reconstructed by parsimony analysis using the Mesquite software (see Methods), and represented as a cladogram in Additional file 4, Figure S1. The proposed evolutionary scenario corresponding to those unshared segments is discussed below, the largest ones first.

The locus of BMI_II545-6 in B. microti CCM 4915 is occupied in the $B$. pinnipedialis $\mathrm{B} 2 / 94$ small chromosome by a 67,389 bp insert (position: 520,136 to 587,524 ) which is present in a number of Brucella strains from marine mammals and partially in B. ovis ATCC 25840. The gene arrangements of this genomic region in $B$. pinnipedialis, B. microti and B. ovis are depicted in Figure 2. This region is flanked on both sides by an IS711 element (respectively BPI_II536-7 and BPI_II602-3), at a location where a sole copy of the IS711 element is found in B. microti CCM 4915 (BMI_II545-6), as in all Brucella strains lacking this insert. This region is partially found in B. ovis ATCC 25840, except in the interval between BPI_II545 and BPI_II576 deleted from B. ovis. The deletion in B. ovis ATCC 25840 occurred inside the gene BOV_A0488, whose 85 first amino acids are similar to BPI_II577 and whose last 100 amino acids are similar to BPI_II544. This interval exhibits a few protein coding genes or their remnants, with similarities mainly found in distant alphaproteobacteria i.e. excluding Brucella and Ochrobactrum. In particular, two pseudogenes, BPI_II545 and BPI_II548, separated by one IS711 element, appear to be the remnants of two consecutive Paracoccus denitrificans genes coding for 5-oxoprolinase (Pden_ 4408-9) and are preceded by an AraC-family transcriptional regulator (BPI_II544) with similarity into the same organism (Pden_4410). The same three genes are also found grouped together but in different orders in the genomes of several betaproteobacteria e.g Bordetella avium (BAV3293-5) or Verminephrobacter eiseniae (Veis_1518-20). Two consecutive genes, etfA (BPI_II555) and etfB (BPI_II556) are also present on the large chromosome with $100 \%$ nucleotide identity. We also observed 4 components of a spermidine/putrescine ABC-transporter (BPI_II564-7) found colinearly in the genomes of e.g. Roseobacter denitrificans (RD1_3862-5) or Silicibacter pomeroyi (SPOA381-4). We found similarities to two consecutive genes of Parvibaculum lavamentivorans (Plav_0386-7), similarities to Plav_0387 being noticed on both sides of the BPI_II574-5 ISBm1 transposase gene. Other genes like the aldehyde dehydrogenase BPI_II571 have best matches in Brucella and Ochrobactrum, but with $73 \%$ similarity only at the amino acid level. Several transposase genes are also present. This large $67 \mathrm{kbp}$ fragment probably found its origin in the common ancestor of the marine mammal Brucella species and B. ovis, as attested by its partial presence in $B$. ovis. The structure of this segment suggests a grouping of $B$. ovis with marine mammal Brucella sp. (Additional file 4, Figure S1-a). It may have been lost thereafter several times, in Brucella sp. strain NVSL 07-006 isolated from a baboon and along the $B$. cet $i$ and $B$. pinnipedialis lineages, putatively by recombination of the two IS711 copies.

A 21,713 bp fragment on the large chromosome (position: 259,190 to 280,902 in B. pinnipedialis B2/94) is not found in B. microti CCM 4915. This fragment is present in all B. melitensis and B. abortus strains and in $B$. neotomae, but its occurrence is quite variable among $B$. ceti and $B$. pinnipedialis strains and even among $B$. suis strains, where it is observed in the earliest diverging B. suis 513 and B. suis ATCC 23445, but absent otherwise. This fragment starts with a phage integrase gene (BPI_I248) and ends with a tRNA (BPI_I278) which was the likely insertion site. An IS711 element is inserted within a gene (BPI_II256) that remained intact in B. melitensis $16 \mathrm{M}$ (BMEI1694) and B. ovis ATCC 25840 (BOV_0245). This fragment encodes a flagellar protein FlgJ (BPI_I260). The other putative genes in this region have no convincing similarities to annotated proteins. This $21 \mathrm{kbp}$ fragment (Additional file 4, Figure S1-b) probably entered the Brucella genomes after $B$. microti divergence, and disappeared separately on several branches. It confirms the grouping of $B$. ceti Cudo, B. ceti B1/94, B. ceti M490/95/1 and Brucella sp. $\mathrm{F} 5 / 99$. Its presence in the genome of $B$. neotomae $5 \mathrm{~K} 33$ and absence in B. suis strains except for B. suis ATCC 23445 and $B$. suis 513 suggest its insertion prior to the 


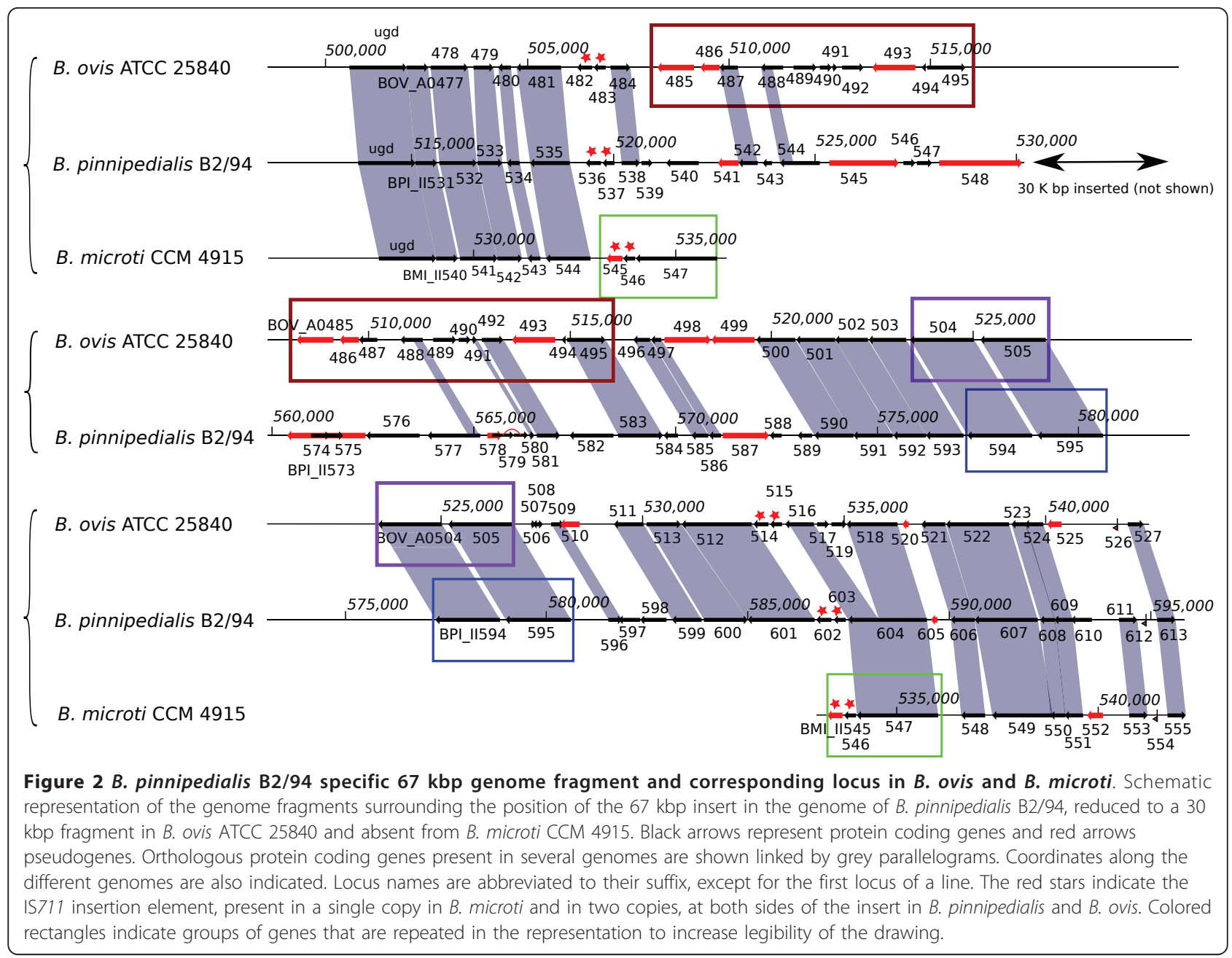

divergence of the $B$. suis and $B$. neotomae lineage, and a subsequent loss in the $B$. suis lineage.

A 18,341 bp region on the small chromosome (between BMI_II357 and BMI_II381) is absent from the B. pinnipedialis B2/94 genome (position: 344,671-363,011 in $B$. microti CCM 4915). This region is present in B. microti, B. suis, B. canis, B. ceti, Brucella sp. F5/99, B. neotomae and absent in B. abortus, B. melitensis, B. ovis and B. pinnipedialis. Additionally, a partial match was found in the genome of Brucella sp. B02. In this region, the presence of genes encoding TraI-J proteins involved in bacterial conjugation can be noted. Like the above $67 \mathrm{kbp}$ fragment, this $18 \mathrm{kbp}$ fragment (Additional file 4, Figure S1c) supports a divergence of the $B$. melitensis/B. abortus clade prior to the separation of B. ovis, Brucella sp. strain NVSL 07-006 and marine mammal Brucella strains. It might have appeared before $B$. microti divergence, and disappeared several times, in particular from the branch leading to the B. abortus/B. melitensis clade and from the branch leading to the $B$. pinnipedialis clade.
The $11 \mathrm{kbp}$ region on the small chromosome is a phage related region discussed in [30] and unique to $B$. microti (position: 1,038,883-1,050,624 in B. microti CCM 4915). It will not be discussed further (Additional file 4, Figure S1-d).

Still on the small chromosome, a 2,881 bp region (position: 1,082,391-1,085,271 in B. microti CCM 4915) encodes genes BMI_II1086-8. This fragment (Additional file 4, Figure S1-e) appeared before B. microti divergence, and is absent from the branch leading to B. ovis and Brucella sp. strain NVSL 07-0026, but also from the branch leading to B. pinnipedialis M292/94/1 and B. pinnipedialis B2/94, separating those two species from $B$. pinnipedialis M163/99/10. Closer examination reveals that this $2.8 \mathrm{kbp}$ fragment is absent in $B$. ovis because it belongs to a much larger $44.5 \mathrm{kbp}$ region deleted from B. ovis [31] and also partially deleted from Brucella sp. NVSL 07-0026, where an approximately $30 \mathrm{kbp}$ long region is missing (pos: $1,117,180$ to 1143394 in B. pinnipedialis B2/94 small chromosome). This finding supports both grouping of the 
B. pinnipedialis M292/94/1 and B2/94 strains, and that of B. ovis with Brucella sp. strain NVSL 07-0026.

Between BMI_I949 and BMI_I953, there is a 2,653 bp region deleted from the $B$. pinnipedialis $\mathrm{B} 2 / 94$ large chromosome (position: 928,716-931,368 in B. microti CCM 4915) but also from B. ovis ATCC 25840, Brucella sp. NVSL 07-0026 and all B. melitensis and B. abortus strains. Interestingly, this deletion occurred inside a gene (encoding an $\mathrm{ABC}$ transporter), thus showing that it is a deletion and not an acquisition event. This region (Additional file 4, Figure S1-f) is particularly informative because it clearly separates Brucella strains into two groups. This fragment was present in ancestral Brucella, and then lost after the divergence of the B. suis clade, of $B$. neotomae, of $B$. microti and prior to the divergence of B. abortus and B. melitensis, B. ovis and Brucella sp. strain NVSL 07-2026, and finally marine mammal Brucella species. An interesting exception is found in $B$. abortus B3196, where this ABC transporter gene is intact. This feature clearly suggests a divergence of the $B$. suis and B. neotomae group before that of the other host-associated Brucella.

\section{IS711 insertion sequences}

IS711 insertion sequences [32] account for a large number of gaps within the complete genome alignment of $B$. pinnipedialis $\mathrm{B} 2 / 94$ and $B$. microti CCM 4915. In total, 19 IS711 elements were identified on the large chromosome and 12 on the small chromosome of B. pinnipedialis. We looked for the occurrence of those insertion sequences in the available complete genomes of Brucella (Methods) and reported their position in Figure 3 and Additional file 5, Table S4. Although it is difficult to analyze transposable repetitive elements in unfinished genomes (as they are often the ones precluding genome assembly) [33], we included two other partial genomes in our analysis, but where an abundant number of IS711 elements were observed, B. ceti Cudo and Brucella sp. strain NVSL 07-0026.

Figure 3 shows that 6 IS711 insertion sequences are found in all selected Brucella strains (IS711 groups numbered $1,4,12,13,26$, and 31 ). We also observed that 4 IS711 elements were uniquely shared by $B$. ovis ATCC 25840 and Brucella sp. strain NVSL 07-006 (groups 41, 43,48 and 54), supporting the grouping of those two species, already noticed when discussing the whole gene tree, and confirmed by the large deletion around the $2.8 \mathrm{kbp}$ fragment discussed previously. Those two species were the likely subjects of an intense IS711 transposition activity, with 14 elements being uniquely found in B. ovis and 9 being uniquely found in Brucella sp. NVSL 07-006. Among the 31 IS711 positions found in B. pinnipedialis B2/94, 21 are shared with $B$. ceti Cudo among which 12 are found only in B. pinnipedialis B2/94 and B. ceti Cudo, and absent from all other strains (groups 5-9, 17, 18, 19, 21, 23 and 24).

\section{Difference in gene content}

Comparing orthologous loci (present in both B. microti CCM 4915 and B. pinnipedialis B2/94 genomes), we found that the number of pseudogenes in the genome of $B$. pinnipedialis $\mathrm{B} 2 / 94$ was larger than that in the genome of B. microti CCM 4915 (30 for B. microti on chromosome I and 48 for B. pinnipedialis, 16 for B. microti on chromosome II and 42 for $B$. pinnipedialis). This was also noticed when comparing B. microti CCM 4915 and B. suis 1330, and was attributed to a slower evolution rate in $B$. microti. The genes that are potentially different in both species are reported in Additional file 6, Table S5.

Among the genes altered in B. pinnipedialis B2/94, many are components of $\mathrm{ABC}$ transporters. Those genes are highlighted in Additional file 6, Table S5. We found 17 genes related to $\mathrm{ABC}$ transporters that are impaired, on a total of 90 impaired genes. In the B. pinnipedialis genome we identified approximately 249 ABC transporter-related intact genes on a total of 3,342 protein coding genes. This output is highly improbable ( $\mathrm{p}$-value $=1 . \mathrm{e}-4$, chi-square test) and strongly suggest that ABC transporters were specifically degraded in B. pinnipedialis and more generally in host-associated Brucella species. Beside ABC type transport systems, many other genes involved in transport are found impaired in B. pinnipedialis B2/94: a CorA family transporter BPI_I592 (ortholog BMI_I558), an EmrB/Qaca family drug resistance transporter BPI_I1098 (ortholog BMI_I1064), the dipeptide transport system permease protein DppC (BPI_I1637, ortholog BMI_I1597), an outer membrane autotransporter BPI_I2072 (ortholog BMI_I2035), a glucose/galactose transporter BPI_II188 (ortholog BMI_II187), a cadmiun-translocating P-type ATPase BPI_II1260 (ortholog BMI_II1204), and finally a putative transport protein BPI_II453 (ortholog BMI_II468).

One of those ABC transporter genes, BPI_I1818, exhibits an interesting feature. A frameshift difference between the B. pinnipedialis B2/94 and B. microti CCM 4915 sequences merges the membrane and ATP-binding components (BMI_I1778-9) of a thiamin ABC transporter into a single reading frame. A blast (tblastn) search of the BPI_I1818 gene sequence against the nucleotide sequences of the other Brucella strains shows that this gene fusion occurred in all marine mammal strains studied, except for B. ceti M13/05/1 and M644/93 which represent the distinct dolphin subgroup of strains within B. ceti mentioned above.

\section{Conclusions}

Time of appearance of marine mammal Brucella species It has been suggested [14] that the divergence of species in the genus Brucella could have been concomitant with 


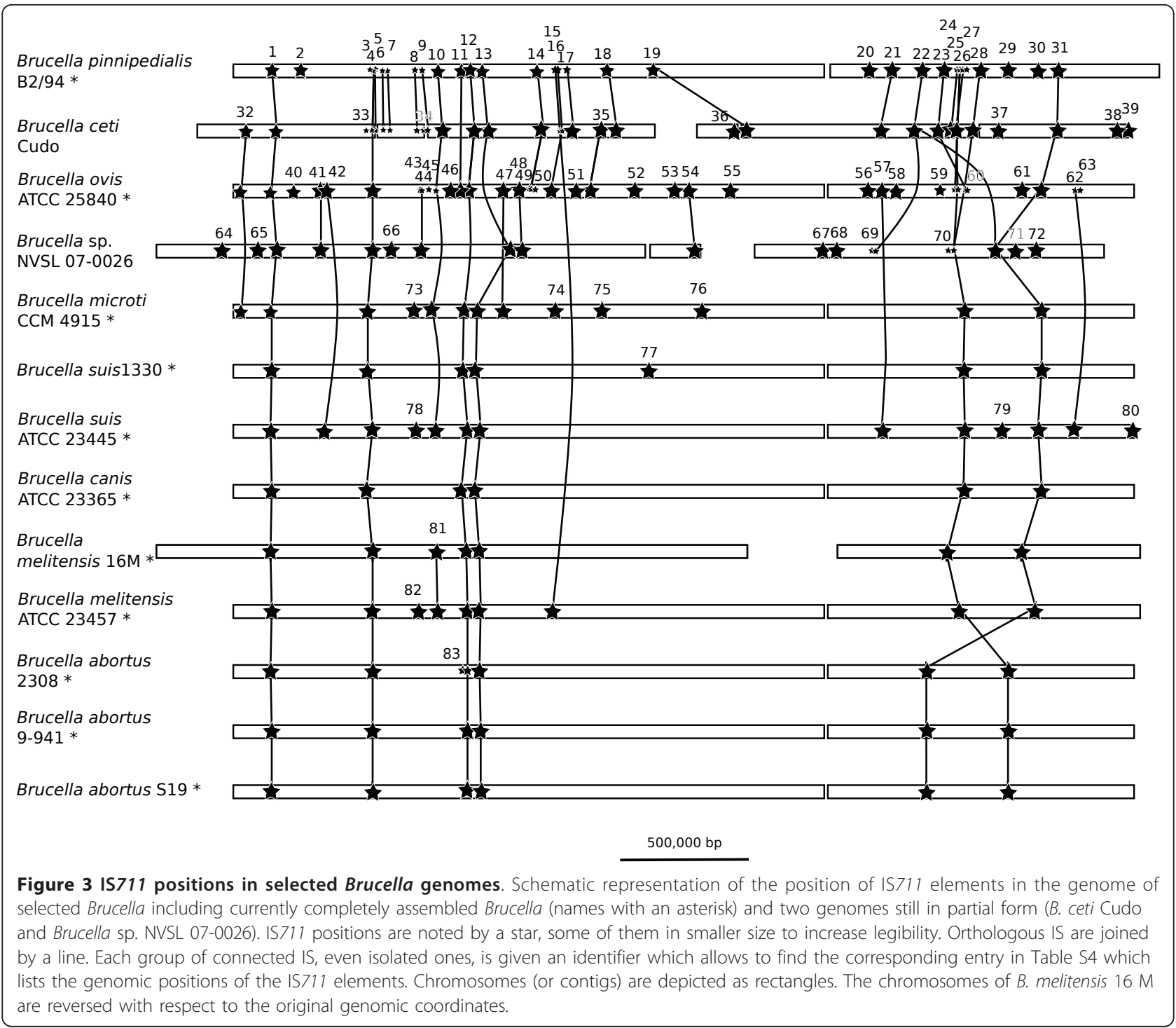

the divergence of their mammalian hosts, 60 millions years (my) ago. However this is inconsistent with the fact that the hosts of $B$. ceti and B. pinnipedialis did not diverge at the same time. The ancestors of pinnipeds where carnivores and Higdon et al. [34] used molecular data to estimate the split between ursids and pinnipeds to $35.7 \pm 2.63(=$ mean $\pm \mathrm{SE}) \mathrm{my}$, and fossil records report early pinnipeds $35 \mathrm{my}$ ago [35]. Cetaceans went back to the sea much earlier, the oldest known cetaceans date back to the Eocene, 55 my ago [36]. If we consider divergence in the $16 \mathrm{~S}$ rRNA gene sequence, and referring to $B$. microti which has a central position, $B$. pinnipedialis, B. melitensis, B. abortus, B. suis (perhaps with the exception of B. suis 513, which has 2 (nt) differences, C$>\mathrm{T}$ at position 11 and G- > T at position 1468), B. ovis, $B$. canis have all identical sequences, and $B$. neotomae has 1 bp difference ( $\mathrm{C}->\mathrm{T}$ at position 541$)$. Using the estimate of $1-2 \%$ of change in $16 \mathrm{~S}$ rRNA sequence per 50 my, 1 bp difference (which really should be considered as a maximal) corresponds to $0.07 \%$ change, and a divergence time of 1.75-3.5 my. This time estimate is probably a crude overestimation and recent work [37], using single nucleotide polymorphisms from 13 genomes, showed that most Brucella species probably diverged 86,000 to 296,000 years ago. This analysis reveals that the divergence time of Brucella sp. found today in marine mammals is totally incompatible with the divergence time of their hosts. A fortuitous contamination of cetaceans and pinnipeds, probably via the food chain, may explain better this transmission of Brucella to the marine mammals. This also opens the remote possibility of marine Brucella infecting terrestrial mammals. 


\section{On the order of appearance of host-associated Brucella species}

The analysis of distinctive genomic regions between B. microti CCM 4915 and B. pinnipedialis B2/94 as well as the study of additional markers reveal the order of appearance of the different Brucella species. It is clearly apparent that most of the events following the divergence of B. microti from the classical Brucella species occurred in a very small amount of time, as if something caused a sudden radiation in this lineage and a subsequent adaptation of the organisms to their hosts. Here we summarize some of the major evolutionary events that highlight the evolutionary history of the genus Brucella.

Following the divergence of B. microti, the next evolutionary event that we can trace is the $2.6 \mathrm{kbp}$ fragment clear disappearance, which tells us that the two next Brucella groups to diverge were B. suis and B. neotomae. We did not find any good marker in favor of a prior divergence of one versus the other.

Marine mammal Brucella species as well as B. ovis and Brucella sp. NVSL 07-0026 share the presence of a high number of IS711 elements, and it has been demonstrated that IS711 transposition is still an active process in B. ovis and B. pinnipedialis [38]. Those insertion elements are much less numerous in B. melitensis and B. abortus strains and we thus assume that IS711 transposition events occurred quite abundantly after the divergence of $B$. melitensis/B. abortus. In this group, $B$. abortus strains share a unique feature which is a genomic $600 \mathrm{kbp}$ inversion in the small chromosome, as clearly depicted on Figure 1 of reference [39].

The whole gene tree, where B. ovis and Brucella sp. NVSL 07-0026 cluster together, the 4 IS711 elements positions that they have in common and not shared with B. pinnipedialis $\mathrm{B} 2 / 94$ as well as the large deletion that those two species share, in the region surrounding the 2.8 kbp deletion in B. pinnipedialis, all those facts support the grouping of B. ovis with Brucella sp. NVSL 07-0026. The grouping of marine mammal Brucella strains that we observe in the tree with early divergence of B. ceti M13/ 05/1 and M644/93 is also reflected by the gene fusion event mentioned earlier. Grouping of B. pinnipedialis M292/94 and B2/94 is supported by the loss of the $18 \mathrm{kbp}$ fragment in these two strains.

\section{The explosive radiation in the genus Brucella}

There is a clear transition in the genus Brucella evolutionary tree. The first Brucella discovered were the host-associated Brucella species, but more recently, B. microti was isolated as the first representative of a fast growing list of free-living Brucella. This biochemically highly active bacteria was found to share more phenotypic traits with Ochrobactrum than with the host-associated Brucella species [6]. We proposed earlier [30] that the transition between a free-living and an host-associated life style could have resulted from the modification in the $23 \mathrm{~S}$ ribosomal RNA gene sequence with putative effects on the growth rate of the bacteria. A slow growth rate has often been advocated for intra-cellular bacteria, as their survival is often dependent on the survival of their hosts [40]. Brucella with this change in $23 \mathrm{~S}$ structure and its impact on growth rate became suddenly more adapted to an hostassociated life style than to a free-living style, and progressively adapted to distinct sets of hosts, giving rise to the main lineages of host-associated Brucella species that are encountered today.

\section{Methods}

\section{Sequencing and origin of sequence data used for comparative work}

Genome was assembled from 430,042 paired GS-FLX reads of average length 229 , giving approximately a $30 \times$ coverage of the genome, and directed sequencing of the remaining gaps was performed using 193 additional Sanger sequencing reactions. The genome sequence is deposited in the complete genome division of GenBank under project ID 41867 and accession numbers CP002078 and CP002079. Genomic sequence data of all Brucella strains mentioned in this work can be conveniently downloaded from a unique location at the Pathosystems Resource Integration Center web site [41], or otherwise from the complete genome and whole genome shotgun divisions of GenBank [42]. Origin of the sequence data is listed in Supplementary Table 3. Most sequences originate from the Brucella group project at the Broad Institute, conducted by Davis O'Callaghan, Adrian Whatmore and Renee Tsolis [43] or from the Pathosystems Resource Integration Center of the Virginia Bioinformatics Institute [41].

\section{Whole gene tree}

Whole gene tree was build using gene sequences from all available Brucella as well as Ochrobactrum intermedium. The procedure used to build the tree is similar to that reported in [30]. Briefly, 1125 orthologous genes from 39 Brucella plus genes from O. intermedium were used. Orthologous genes were selected using the following procedure. A file containing nucleotide gene sequences for the selected organisms was compared to itself using blastn [44] (parameters: -b 100 -v 100 -F F -e 1.e-20). The resulting output file was subjected to clustering using the Markov chain clustering algorithm [45]. In the resulting cluster list, we selected the clusters with only one unique member per species. Genes from each cluster were then aligned using MUSCLE [46] (default parameters). The following alignments were concatenated, resulting in an alignment of 40 sequences, with 1,078,083 positions, cleaned with Gblock [47] (default parameters), which 
reduced it to 945,578 positions. From this multiple alignment, on which 767,738 sites without polymorphisms and 2189 distinct patterns were found, a tree was inferred by maximum likelihood using PhyML [48], with 100 bootstrap replicates.

\section{Unshared genome segments, tracing segments history}

The nucleotide sequence of the genome fragment coming either from B. pinnipedialis B2/94 or B. microti CCM 4915 was used as a query for blastn search (e-value $=1$.e100, no filter) against the nucleotide sequences of the remaining Brucella. For each target genome, the number of distinct nucleotide positions that had a hit was recorded in Additional file 3, Table S3. Presence or absence of a given fragment is represented in Figure 1 alongside the whole gene tree. The history of those genome segments was subsequently traced using the Mesquite software package [49] on cladograms reflecting the topology obtained from the whole gene tree (Figure 1). Character history was computed using parsimony analysis, with presence or absence of a genome segment treated as a discrete category character.

\section{Across genomes identification of IS711 element positions} A database containing the complete genomes of the Brucella species under study was compiled. We searched this database for the occurrence of IS711 using the nucleotide sequence of one element from B. pinnipedialis as query, using the blastn program (parameters: -b 1000 -v 1000, limiting to hits with a score of 500). We extracted the corresponding segments, adding 500 nucleotides of context on both side. We then masked this file so that the IS711 sequence itself was replaced by $x$ in the sequence. A subsequently blastn search of this file against itself allowed us to recover the orthologous IS711 positions along the different Brucella genomes (listed in Additional file 5, Table S4), used for the representation in Figure 3.

\section{Additional material}

Additional file 1: Indels in B. pinnipedialis B2/94 and B. microti large chromosome alignment. List of insertions and deletions (indels) in the complete genome alignment of B. pinnipedialis B2/94 and B. microti CCM 4915, large chromosome

Additional file 2: Indels in B. pinnipedialis B2/94 and B. microti small chromosome alignment. List of insertions and deletions (indels) in the complete genome alignment of B. pinnipedialis B2/94 and B. microti CCM 4915, small chromosome.

Additional file 3: Occurrences of the largest genome fragments unshared between B. pinnipedialis B2/94 and B. microti CCM 4915 in selected Brucella genomes. Occurrences of the largest genome fragments unshared between B. pinnipedialis B2/94 and B. microti CCM 4915 in selected Brucella genomes. For each organism, the number of positions in its genome that are found similar to the original fragment is reported. The column "Origin of sequence data" indicates "GenBank" for sequences deposited in the complete genome division of GenBank,
"Patric" if the sequence originates from the Pathosystems Resource Integration Center of the Virginia Bioinformatics Institute, and "Broad" if he sequence originates from the Brucella genome sequencing project at the Broad Institute.

Additional file 4: Evolutionary history of the unshared sequence fragments along the Brucella evolutionary tree. Evolutionary history of the unshared sequence fragments along the Brucella evolutionary tree. The tree is represented as a cladogram with the same topology as that of the whole gene tree (Figure 1). Plots are generated using Mesquite.

Additional file 5: Position of IS711 insertion sequences in the genome of completely sequences Brucella, $B$. ceti Cudo and Brucella sp. NVSL 07-0026. Position of IS711 insertion sequences in the genome of completely sequences Brucella, B. ceti Cudo and Brucella sp. NVSL 070026. Each IS711 element position as well as the accession number of the genome segment to which it belongs are listed in the Table. Clusters of IS711 elements are identified by a number allowing to locate them in Figure 3.

Additional file 6: List of genes showing a large change between $B$. pinnipedialis B2/94 and B. microti CCM 4915. List of genes showing a large change between B. pinnipedialis B2/94 and B. microti CCM 4915. $A B C$ transporter genes are indicated.

\section{Author details}

'Laboratoire Information Génomique et Structurale, CNRS-UPR2589, AixMarseille University, Institut de Microbiologie de la Méditerranée (IM2, IFR88), Parc Scientifique de Luminy-163 Avenue de Luminy-Case 934-FR-13288, Marseille cedex 09, France. ${ }^{2}$ CNRS, UMR 7144, Equipe Evolution du Plancton et Paléo-Océans, Station Biologique de Roscoff, 29682 Roscoff, France. ${ }^{3}$ UPMC Univ Paris 06, UMR 7144, Adaptation et Diversité en Milieu Marin, Station Biologique de Roscoff, 29682 Roscoff, France. ${ }^{4}$ INRA, UR1282, Infectiologie Animale et Santé Publique, IASP, Nouzilly, F-37380, France.

\section{Authors' contributions}

SA participated in the design of the study, performed genome assembly, annotation and comparisons with other Brucella and wrote the manuscript. ML participated in genome annotation and analyzed the data. JMC participated in the design of the study, analyzed data and wrote the manuscript. MZ and AC designed the study, coordinated the research, analyzed data and wrote the manuscript. All authors read and approved the final manuscript.

Received: 1 April 2011 Accepted: 11 July 2011 Published: 11 July 2011

\section{References}

1. Godfroid J, Cloeckaert A, Liautard JP, Kohler S, Fretin D, Walravens K, GarinBastuji B, Letesson JJ: From the discovery of the Malta fevers agent to the discovery of a marine mammal reservoir, brucellosis has continuously been a re-emerging zoonosis. Vet Res 2005, 36:313-326.

2. Moreno E, Cloeckaert A, Moriyón I: Brucella evolution and taxonomy. Vet Microbiol 2002, 90:209-227.

3. Whatmore AM: Current understanding of the genetic diversity of Brucella, an expanding genus of zoonotic pathogens. Infect Genet Evol 2009, 9:1168-1184

4. Foster G, MacMillan AP, Godfroid J, Howie F, Ross HM, Cloeckaert A, Reid RJ, Brew S, Patterson IAP: A review of Brucella sp. infection of sea mammals with particular emphasis on isolates from Scotland. Vet Microbiol 2002, 90:563-580.

5. Foster G, Osterman BS, Godfroid J, Jacques I, Cloeckaert A: Brucella ceti sp. nov. and Brucella pinnipedialis sp. nov. for Brucella strains with cetaceans and seals as their preferred hosts. Int I Syst Evol Microbiol 2007, 57:2688-2693.

6. Scholz HC, Hubalek Z, Sedlácek I, Vergnaud G, Tomaso H, Al Dahouk S, Melzer F, Kämpfer P, Neubauer H, Cloeckaert A, Maquart M, Zygmunt MS, Whatmore AM, Falsen E, Bahn P, Göllner C, Pfeffer M, Huber B, Busse HJ, Nöckler K: Brucella microti sp. nov., isolated from the common vole Microtus arvalis. Int I Syst Evol Microbiol 2008, 58:375-382.

7. Scholz HC, Hofer E, Vergnaud G, Le Fleche P, Whatmore AM, Al Dahouk S, Pfeffer M, Krüger M, Cloeckaert A, Tomaso H: Isolation of Brucella microti 
from Mandibular Lymph Nodes of Red Foxes, Vulpes vulpes, in Lower Austria. Vector Borne Zoonotic Dis 2009, 9:153-156.

8. Scholz HC, Hubalek Z, Nesvadbova J, Tomaso H, Vergnaud G, Le Flèche P, Whatmore AM, Al Dahouk S, Krüger M, Lodri C, Pfeffer M: Isolation of Brucella microti from soil. Emerg Infect Dis 2008, , 14: 1316-1317.

9. De BK, Stauffer L, Koylass MS, Sharp SE, Gee JE, Helsel LO, Steigerwalt AG, Vega R, Clark TA, Daneshvar MI, Wilkins PP, Whatmore AM: Novel Brucella strain (BO1) associated with a prosthetic breast implant infection. J Clin Microbiol 2008, 46:43-49.

10. Scholz HC, Nöckler K, Göllner C, Bahn P, Vergnaud G, Tomaso H, Al-Dahouk S, Kämpfer P, Cloeckaert A, Maquart M, Zygmunt MS, Whatmore AM, Pfeffer M, Huber B, Busse HJ, De BK: Brucella inopinata sp. nov., isolated from a breast implant infection. Int J Syst Evol Microbiol 2010, 60:801-808.

11. Schlabritz-Loutsevitch NE, Whatmore AM, Quance CR, Koylass MS, Cummins LB, Dick EJ Jr, Snider CL, Cappelli D, Ebersole JL, Nathanielsz PW, Hubbard GB: A novel Brucella isolate in association with two cases of stillbirth in non-human primates-first report. J Med Primatol 2009, 38:70-73.

12. Tiller RV, Gee JE, Frace MA, Taylor TK, Setubal JC, Hoffmaster AR, De BK: Characterization of novel Brucella strains originating from wild native rodent species in North Queensland, Australia. Appl Environ Microbiol 2010, 76:5837-5845.

13. Tiller RV, Gee JE, Lonsway DR, Gribble S, Bell SC, Jennison AV, Bates J, Coulter C, Hoffmaster AR, De BK: Identification of an unusual Brucella strain (BO2) from a lung biopsy in a 52 year-old patient with chronic destructive pneumonia. BMC Microbiology 2010, 10:23.

14. Bourg G, OCallaghan D, Boschiroli ML: The genomic structure of Brucella strains isolated from marine mammals gives clues to evolutionary history within the genus. Vet Microbiol 2007, 125:375-380.

15. Clavareau C, Wellemans V, Walravens K, Tryland M, Verger JM, Grayon M, Cloeckaert A, Letesson JJ, Godfroid J: Phenotypic and molecular characterization of a Brucella strain isolated from a minke whale (Balaenoptera acutorostrata). Microbiology 1998, 144:3267-3273.

16. Cloeckaert A, Verger JM, Grayon M, Paquet JY, Garin-Bastuji B, Foster G, Godfroid J: Classification of Brucella spp. isolated from marine mammals by DNA polymorphism at the omp2 locus. Microbes Infect 2001, 3:729-738.

17. Dawson CE, Stubberfield EJ, Perrett LL, King AC, Whatmore AM, Bashiruddin JB, Stack JA, Macmillan AP: Phenotypic and molecular characterisation of Brucella isolates from marine mammals. BMC Microbiol 2008, 8:224.

18. Groussaud P, Shankster SJ, Koylass MS, Whatmore AM: Molecular typing divides marine mammal strains of Brucella into at least three groups with distinct host preferences. J Med Microbiol 2007, 56:1512-1518.

19. Maquart M, Le Flèche P, Foster G, Tryland M, Ramisse F, Djønne B, Al Dahouk S, Jacques I, Neubauer H, Walravens K, Godfroid J, Cloeckaert A, Vergnaud G: MLVA-16 typing of 295 marine mammal Brucella isolates from different animal and geographic origins identifies 7 major groups within Brucella ceti and Brucella pinnipedialis. BMC Microbiol 2009, 9:145.

20. McDonald WL, Jamaludin R, Mackereth G, Hansen M, Humphrey S, Short P, Taylor T, Swingler J, Dawson CE, Whatmore AM, Stubberfield E, Perrett LL, Simmons G: Characterization of a Brucella sp. strain as a marine-mammal type despite isolation from a patient with spinal osteomyelitis in New Zealand. J Clin Microbiol 2006, 44:4363-4370.

21. Sohn AH, Probert WS, Glaser CA, Gupta N, Bollen AW, Wong JD, Grace EM, McDonald WC: Human neurobrucellosis with intracerebral granuloma caused by a marine mammal Brucella spp. Emerg Infect Dis 2003, 9:485-488.

22. Whatmore AM, Dawson CE, Groussaud P, Koylass MS, King AC, Shankster SJ, Sohn AH, Probert WS, McDonald WL: Marine mammal Brucella genotype associated with zoonotic infection. Emerg Infect Dis 2008, 14:517-518

23. Halling SM, Tatum FM, Bricker BJ: Sequence and characterization of an insertion sequence, IS711, from Brucella ovis. Gene 1993, 133:123-127.

24. Ouahrani S, Michaux S, Sri Widada J, Bourg G, Tournebize R, Ramuz M Liautard JP: Identification and sequence analysis of IS6501, an insertion sequence in Brucella spp.: relationship between genomic structure and the number of IS6501 copies. J Gen Microbiol 1993, 139:3265-3273.

25. Bricker BJ, Ewalt DR, MacMillan AP, Foster G, Brew S: Molecular characterization of Brucella strains isolated from marine mammals. J Clin Microbiol 2000, 38:1258-1262.

26. Cloeckaert A, Grayon M, Grépinet O, Boumedine KS: Classification of Brucella strains isolated from marine mammals by infrequent restriction site-PCR and development of specific PCR identification tests. Microbes Infect 2003, 5:593-602.
27. Maquart M, Fardini Y, Zygmunt MS, Cloeckaert A: Identification of nove DNA fragments and partial sequence of a genomic island specific of Brucella pinnipedialis. Vet Microbiol 2008, 132:181-189.

28. Zygmunt MS, Maquart M, Bernardet N, Doublet B, Cloeckaert A: Novel IS711-specific chromosomal locations useful for identification and classification of marine mammal Brucella strains. J Clin Microbiol 2010, 48:3765-3769.

29. Bohlin J, Snipen L, Cloeckaert A, Lagesen K, Ussery D, Kristoffersen AB, Godfroid J: Genomic comparisons of Brucella spp. and closely related bacteria using base compositional and proteome based methods. BMC Evol Biol 2010, 10:249.

30. Audic S, Lescot M, Claverie JM, Scholz HC: Brucella microti: the genome sequence of an emerging pathogen. BMC Genomics 2009, 10:352.

31. Tsolis RM, Seshadri R, Santos RL, Sangari FJ, Lobo JM, de Jong MF, Ren Q, Myers G, Brinkac LM, Nelson WC, Deboy RT, Angiuoli S, Khouri H, Dimitrov G, Robinson JR, Mulligan S, Walker RL, Elzer PE, Hassan KA, Paulsen IT: Genome degradation in Brucella ovis corresponds with narrowing of its host range and tissue tropism. PLOS ONE 2009, 4:e5519.

32. Siguier P, Perochon J, Lestrade L, Mahillon J, Chandler M: ISfinder: the reference centre for bacterial insertion sequences. Nucleic Acids Res 2006, 34D:32-36.

33. Lerat E: Identifying repeats and transposable elements in sequenced genomes: how to find your way through the dense forest of programs. Heredity 2010, 104:520-533.

34. Higdon J, Bininda-Emonds O, Beck R, Ferguson S: Phylogeny and divergence of the pinnipeds (Carnivora: Mammalia) assessed using a multigene dataset. BMC Evol Biol 2007, 7:216.

35. Rybczynski N, Dawson MR, Tedford RH: A semi-aquatic Arctic mammalian carnivore from the Miocene epoch and origin of Pinnipedia. Nature 2009, 458:1021-1024

36. Thewissen JGM, Cooper LN, Clementz MT, Bajpai S, Tiwari BN: Whales originated from aquatic artiodactyls in the Eocene epoch of India. Nature 2007, 450:1190-1194

37. Foster JT, Beckstrom-Sternberg SM, Pearson T, Beckstrom-Sternberg JS, Chain PS Roberto FF, Hnath J, Brettin T, Keim P: Whole Genome-Based Phylogeny and Divergence of the Genus Brucella. J Bacteriol 2009, 191:2864-2870.

38. Ocampo-Sosa A, Garcia-Lobo J: Demonstration of IS711 transposition in Brucella ovis and Brucella pinnipedialis. BMC Microbiol 2008, 8:17.

39. Wattam AR, Williams KP, Snyder EE, Almeida NF Jr, Shukla M, Dickerman AW, Crasta OR, Kenyon R, Lu J, Shallom JM, Yoo H, Ficht TA, Tsolis RM, Munk C, Tapia R, Han CS, Detter JC, Bruce D, Brettin TS, Sobral BW, Boyle SM, Setubal JC: Analysis of ten Brucella genomes reveals evidence for horizontal gene transfer despite a preferred intracellular lifestyle. J Bacterio/ 2009, 191:3569-3579.

40. Winkler HH: Rickettsia prowazekii, ribosomes and slow growth. Trends Microbiol 1995, 3:196-198.

41. Pathosystems resource integration center. [http://brcdownloads.vbi.vt. edu/patric2/PATRIC/.

42. National Center for Biotechnology Information. [http://www.ncbi.nlm.nih. gov].

43. Brucella group project at the Broad Institute. [http://www.broadinstitute. org/annotation/genome/brucella_group/lnfo.html].

44. Altschul SF, Madden TL, Schäffer AA, Zhang J, Zhang Z, Miller W, Lipman DJ: Gapped BLAST and PSI-BLAST: a new generation of protein database search programs. Nucleic Acids Res 1997, 25:3389-33402.

45. Enright AJ, Van Dongen S, Ouzounis CA: An efficient algorithm for largescale detection of protein families. Nucleic Acids Res 2002, 30:1575-1584.

46. Edgar RC: MUSCLE: multiple sequence alignment with high accuracy and high throughput. Nucleic Acids Res 2004, 32:1792-1797.

47. Castresana J: Selection of conserved blocks from multiple alignments for their use in phylogenetic analysis. Mol Biol Evol 2000, 17:540-552.

48. Guindon S, Gascuel O: A simple, fast, and accurate algorithm to estimate large phylogenies by maximum likelihood. Syst Biol 2003, 52:696-704.

49. Mesquite: a modular system for evolutionary analysis. [http:// mesquiteproject.org].

doi:10.1186/1471-2148-11-200

Cite this article as: Audic et al: The genome sequence of Brucella pinnipedialis B2/94 sheds light on the evolutionary history of the genus Brucella. BMC Evolutionary Biology 2011 11:200. 